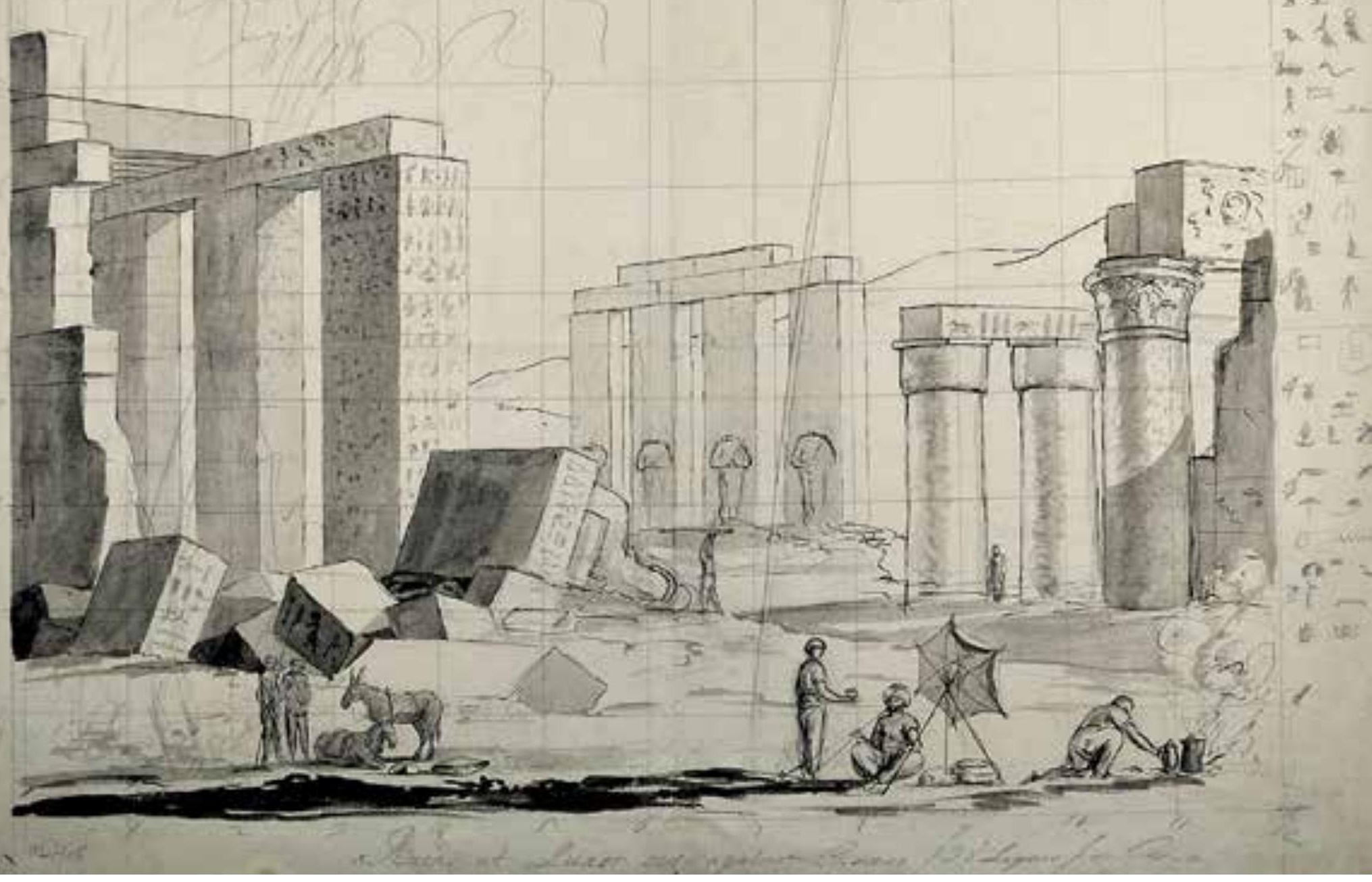

Carl Adolph Gottlob von Schachmann (?): Ansicht des Tempels von Luxor, Pinsel in Wasserfarben und Feder in Tusche über Graphit Kulturhistorisches Museum Görlitz, OLH 18 - unverzeichnet Foto: Kai Wenzel

Carl Adolph Gottlob von Schachmann: Exlibris, Sphinx mit dem Wappen der Familie von Schachmann, Radierung, undatiert Kulturhistorisches Museum Görlitz Foto: Kai Wenzel

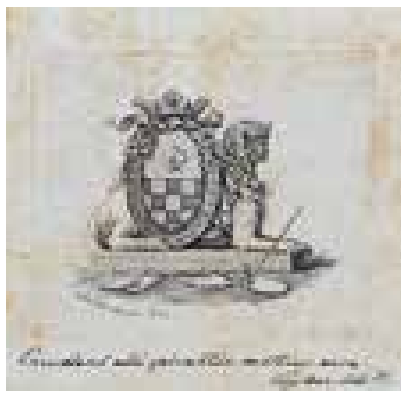

\title{
Carl Adolph Gottlob von Schachmann (1725-1789) und der Orient
}

\author{
Rüdiger Kröger
}

Das Thema mag überraschen, denn dafür, dass der weit gereiste Erbauer des Schlosses Königshain, Carl Adolph Gottlob vom Schachmann ${ }^{1}$, selbst im Orient gewesen sei, ist bisher keine schriftliche Quelle bekannt. Auch seine Bibliothek, enthielt - soweit dies rekonstruierbar ist - nur zwei Werke explizit zu ägyptischen Themen. ${ }^{2}$ Und dennoch muss Schachmann eine Leidenschaft für den Orient, insbesondere Ägypten, entwickelt haben. Sichtbares Zeichen dafür ist sein selbst radiertes, vermutlich in den 1770er Jahren entstandenes Exlibris. Originellerweise dient dem Oberlausitzer Adeligen nicht etwa ein Löwe, sondern ein Sphinx als Schildhalter seines Wappens.

Schachmanns Beziehung zu Ägypten reicht dabei etwa 20 Jahre weiter zurück. In seinem Herrnhuter Umfeld ergab sich nämlich die Möglichkeit, mit Personen in Verbindung zu kommen, die selbst in Ägypten gewesen waren.
So hielt sich z. B. 1751/52 der Admiral, Orientreisende und Abenteurer Pierre Joseph Le Roux, Comte Desneval $(\dagger \text { 1757? })^{3}$ in Herrnhut bzw. Berthelsdorf auf. Er hatte bereits zuvor mit Zinzendorf in London Pläne für eine Reise nach Abessinien (Äthiopien) geschmiedet.

Dann schmarotzerte er bei den vornehmen Mitgliedern der Brüdergemeine in Herrnhut, wo er teilweise wohlwollende Unterstützung, ja Begeisterung fand, teilweise auf Ablehnung stieß. Desneval war auch in Abwesenheit Tischgespräch, wie aus dem Tagebuch der mit Schachmann befreundeten Sowphie Auguste von Schrautenbach hervorgeht: „Abends hatten wir den Carles bey uns zu Tische. Der Discourss war immer von D’Esnevalls.“4

Bereits 1750 war der Herrnhuter Bruder und Arzt Friedrich Wilhelm Hocker (1713-1782) von einer mehrjährigen Missionsreise in den Orient (Syrien, Persien, Ägypten) zurückge- 


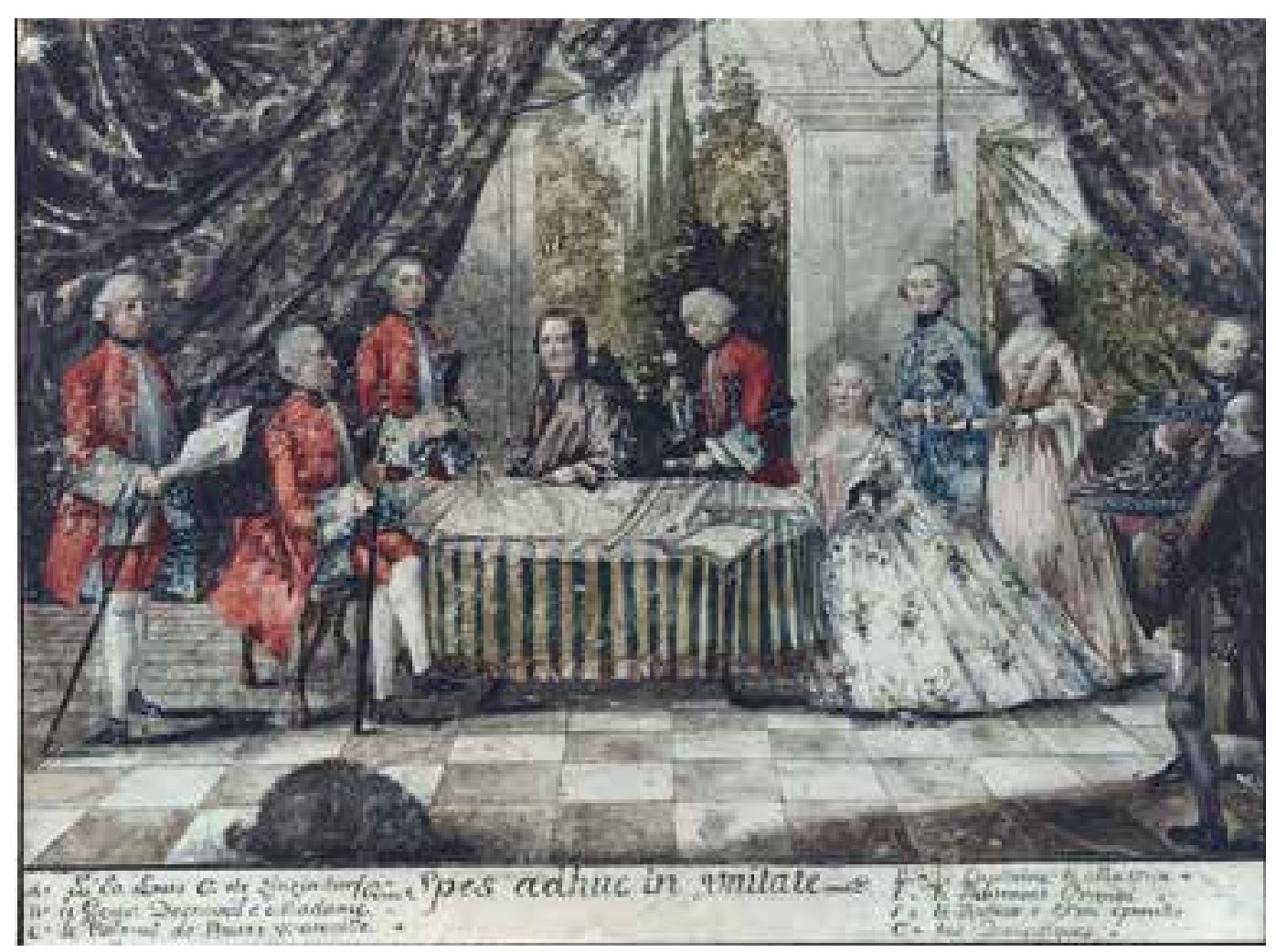

kehrt. ${ }^{5}$ Er dürfte Schachmann mit den ersten verlässlichen Berichten aus dem Orient versorgt haben. Hocker betreute auch Schachmanns todkranke Frau, konnte ihr aber medizinisch nicht mehr helfen. 1752 bis 1755 war er erneut in Kairo tätig. Dort traf er wieder mit Desneval zusammen und hat mit ihm einige leidvolle Erfahrungen machen müssen. ${ }^{6}$ Bei seiner erneuten Rückkehr wusste er manch seltsame Dinge davon zu berichten, wie etwa bei einem im Tagebuch des Grafen Heinrich XXXI. Reuß dokumentierten Spaziergang in nobler Gesellschaft:

„Ich und Heinrich XXVIII. schlugen einen Spa-

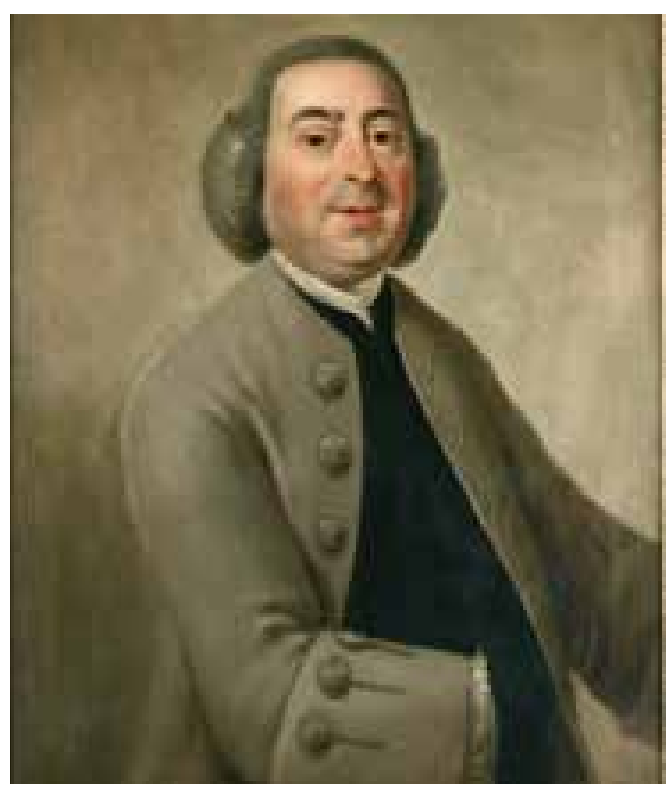

ziergang vor und nahmen Nicolas Watteville und Charles [Schachmann] mit: wir gingen durch den Herrschaftsgarten, herunter ins Tal und so zu den Sieben Stücken [Wiesen] [...] In den Sieben Stücken trafen wir Hocker und Luedeke und vergnügten uns bei dem lieblichen Spaziergang, dem hierherum keiner gleicht. Wir redeten viel vom Grafen Desneval, und Hocker erzählte hässliche Dingen von ihm."7

In Ägypten waren es die Herrnhuter gewöhnt, die landesübliche Kleidung zu tragen. „Denn es komt den Leuten hier sehr wunderlich vor, wenn sie jemand in europäischer Kleidung sehen." heißt es im offiziellen Kairoer Tagebuch. ${ }^{8}$ Hocker brachte, wie später auch andere, seine ,türkischen' Trachten aus Ägypten mit. Es verbreitete ein exotisches Vergnügen, wenn sich Hocker dann und wann ,türkisch' kleidete und auch seine Freunde entsprechend ausstaffierte. Einmal war Hocker mit einem jüngeren ledigen Bruder namens Benjamin Latrobe (1728-1786) in dieser Verkleidung zu Gast bei Zinzendorf, der mitanwesende getaufte Türke Ernst Albert Carl Christiani $(† 1784)^{9}$ machte dazu einen guten türkischen Kaffee. ${ }^{10}$ Etwa ein Jahr später waren Hocker und Latrobe wieder verkleidet, diesmal im Herrnhuter Brüderhaus und Schachmann war diesmal mit von der Partie:

„Hocker lud uns zu einem türkischen, das heißt, einem sehr guten Kaffee ein. Er selbst hatte seine besten türkischen Kleider angelegt und seinen Doktorhut aufgesetzt, Charles [Schachmann] seine zweitbesten, und er trug einen Schnurrbart. Benny [Latrobe] zog die Reste von
Unbekannter Künstler: Zinzendorf und Desneval studieren 1751 in London Landkarten von Abessinien, um 1752 Unitätsarchiv Herrnhut, GS 579
Christian Ludwig Krügelstein: Friedrich Wilhelm Hocker (1713-1782), Arzt in Kairo, 1768 Unitätsarchiv Herrnhut, GS 383 


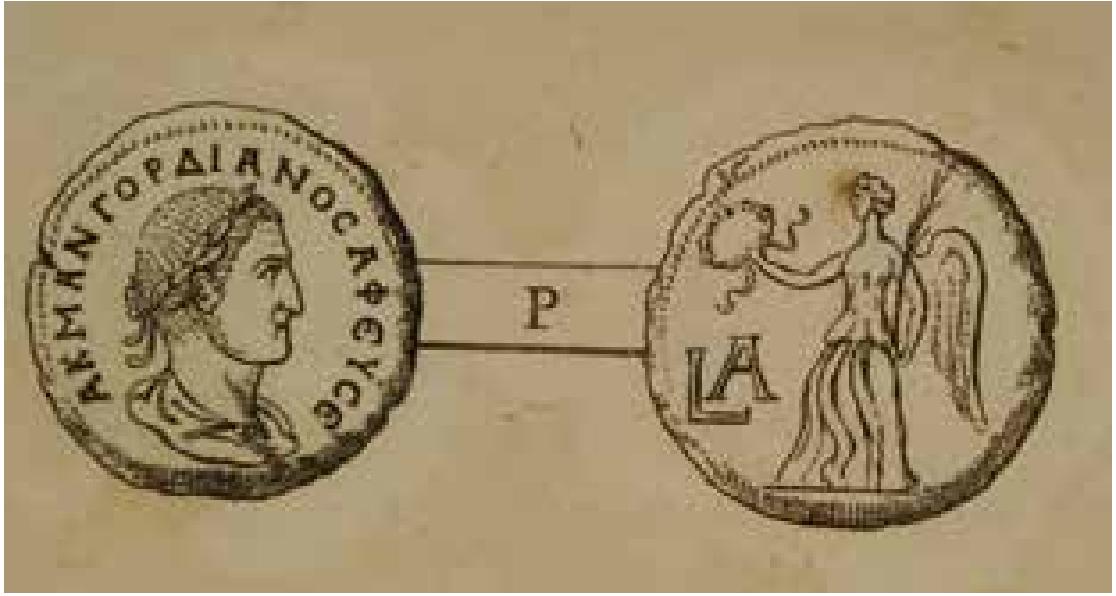

Carl Adolph Gottlob von Schachmann: Münze des Kaisers Gordian filius (ca.192-238), aus einem Hortfund (1760) in Oberägypten aus: Schachmann, Catalogue raisonné..., Leipzig 1774, S. 118

Hockers Kleidern an. Charles sah großartig aus und sehr fein, das volle, runde Gesicht und der kurze Hals passten wunderbar zum türkischen Kostüm, sein Schnurrbart veränderte sein Gesicht so sehr, dass viele Brüder bei ihrem Leben nicht sagen konnten, wer es sei. Und [selbst] Friedrich Watteville erkannte ihn überhaupt nicht. Eine große Versammlung kam dann in Andresens Stube zusammen, probierte den türkischen Kaffee, und nur Benny [Latrobe] war traurig gestimmt, weil er so eine schlechte Figur machte, so dass Hocker ihn [schließlich] einen Türken nannte, der ein Christ werden würde. Hocker ging dann im Haus in allen Stuben herum und verbreitete viel Freude unter den Brüdern. Einige Brüder standen ehrfürchtig von ihren Plätzen auf und nahmen ihre Mützen ab vor dem fremden Herrn, den sie - aus der Nähe betrachtet - nur allzu gut erkannten.“11

Hocker war es denn wohl auch, der von einem weiteren Aufenthalt in Ägypten (17571761) archäologische Objekte für Schachmann mitbrachte. Ein Hauptinteresse Schachmanns bestand in der antiken Numismatik. Seine überschaubare, aber besonders exquisite Sammlung dokumentierte Schachmann in einem von ihm selbst angefertigten und mit eigenen Radirungen illustrierten $\mathrm{Ka}$ talog (1774). ${ }^{12}$ Nur selten gibt Schachmann genauere Angaben über Fundorte oder Erwerbungszusammenhänge. Die Tatsache aber, dass dies gerade für 1759 und 1760 in Ägypten entdeckte Münzen aus der Nähe von Kairo bzw. Oberägypten der Fall ist, erlaubt die Vermutung eins Reisemitbringsels von Hocker, ohne das bisher ein direkter Beweis gelungen wäre. 1765 war Schachmann jedenfalls im Besitz einer dieser Münzen. Das geht aus einem Brief des Direktors der Antikensammlung in Dresden, Johann Friedrich Wacker, hervor, in welchem er nicht nur die Zuschreibung der Münze diskutiert, sondern sich auch für vier kleine Antiken bedankt, die Schachmann, als einer der ersten privaten Förderer, der kurfürstlichen Sammlung geschenkt hatte. Es handelte sich dabei um eine Bronzefigur der den Horus stillenden Isis und drei kleineren Porzellanfiguren, die als „Isis averrunca“, d.h. der die bösen Geister vertreibenden Isis bezeichnet wurden. ${ }^{13}$ Wacker bedankt sich darüber hinaus für ein persönliches Geschenk: „Vor die mir guttigst geschenckten Müntzen sage unterthänigen Dank, die etlichen nummo aegyptiacae fabricas sind mir willckommen geweßen, weil ich bis daher noch nicht einen einzigen dieser Art habhafft werden können." 14

Als Nathanel Gottfried Leske 1782 Schachmann in Königshain besuchte, sah er nicht nur „verschiedene ser seltene egyptische Idolen von Bronze und der sogenanten Porzellanerde“15, sondern auch „verschiedene Arten von Granit aus Egypten. Kalkstein, ebendaher. Muschelmarmor, von den Piramiden bei Gize"16. Wann und von wem Schachmann diese erwarb, ist unbekannt. Die knappe Beschreibung der Idole entspricht derjenigen von Objekten, die Schachmann fast zwanzig Jahre zuvor der Galerie in Dresden geschenkt hatte. Schachmann muss also eine ganze Anzahl ähnlicher Stücke besessen haben. Die Gesteine aus Ägypten möchten aber vielleicht erst kurz zuvor erworben worden sein, weil sich Schachmann vermutlich erst ab 1779 mit der Geologie, insbesondere seiner Königshainer Berge, befasst hatte. ${ }^{17}$

Nach Gründung der Oberlausitzischen Gesellschaft der Wissenschaften bereicherte Schachmann auch deren Kuriositäten- und Naturalienkabinett durch verschiedene Schenkungen, unter anderem mit Ethnographica aus den Missionsgebieten der Brüdergemeine. Für das Jahr 1780 registriert die Zugangsliste „Ein türkisches Schreibzeug von Siegelerde. Geschenk von Schachmann". ${ }^{18}$ Dabei könnte es sich ebenfalls um in Objekt aus Ägypten gehandelt haben. In einer zeitgenössischen Publikation findet sich für den Gebrauch eines solchen „Schreibzeuges" eine anschauliche Beschreibung.

„In Constantinopel, Smirna, Aegypten, Syrien und Palästina habe ich gefunden, daß die Schreiber so wohl vornehmer Herren, als auch der Gesetze, die Schreibmeister der Schüler, wie auch viele Kaufleute, ihr Schreibzeug, als das Dintenfaß und das Pennal, hinter dem Gurt, womit sie ihren Unterrock bey den Hüften zusammengürten, anstecken. In dem Busen haben sie ein ledernes Behältniß des Papiers. Wenn sie nun etwas zu schreiben haben, so nehmen sie aus dem Busen Papier, ergreifen die Feder, öffnen das Dintenfaß, welches hinter dem Gurt stecken bleibet, legen das Papier auf ihre linke Hand, wie wir auf den Schreibtisch, und schreiben also."19

Für die Frage, wie Schachmann an diese Objekte gelangt sein mag, ist man vorerst auf Vermutungen angewiesen. Zuerst ist an die Möglich- 


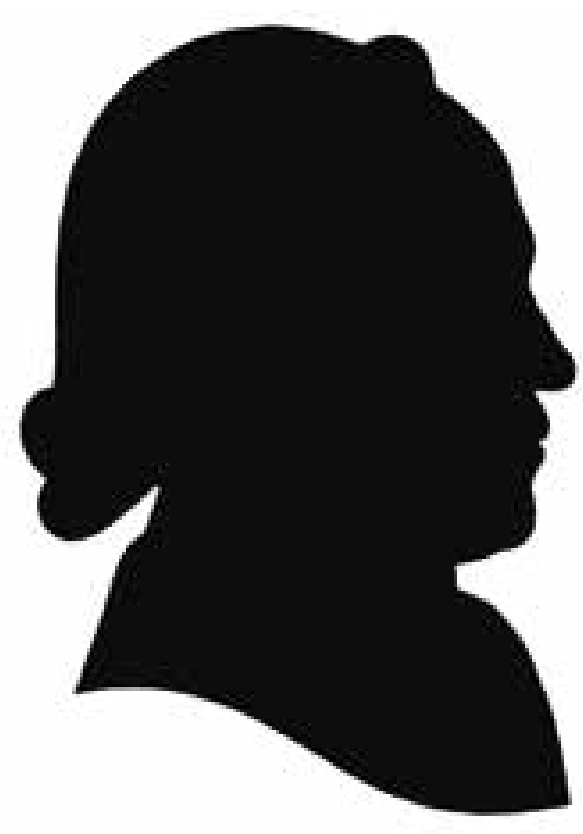

keit $\mathrm{zu}$ denken, dass es sich um persönliche „Mitbringsel“ von Herrnhuter Missionaren handeln kann. Hocker ist, wie bereits angedeutet nicht wieder aus Ägypten zurückgekehrt; wie etliche andere der ausgesandten Herrnhuter Brüder - Frauen wurden in das islamische Land nicht entsandt - verstarb er in Ägypten. Kurze Zeit zuvor war John Antes (1740-1811) nach zwölfjährigem Dienst in Ägypten aufgebrochen und kam 1782 nach Herrnhut zurück, um der Synode einen persönlichen Bericht über den Stand der Arbeit zu liefern. Wie er in seinen 1801 in deutscher Übersetzung erschienenen ,Beobachtungen über die Sitten und Gebräuche der Ägypter' an zwei unterschiedlichen Stellen mitteilt, hat er Naturalien aus Ägypten mit nach Sachsen gebracht. Er erwähnt zunächst eine Probe vom Nilwasser, das angeblich niemals in Fäulnis geriete: „Ich habe selbst eine kleine Flasche voll davon mit nach Europa gebracht, die ich in einem Naturalienkabinet in Sachsen gelassen habe, und das nie einige Spuren von Gährung zeigte. ${ }^{20}$ Das zweite Objekt, welches er nennt, ist ein ausgestopftes Krokodil von beachtlicher Größe. Bei dieser Gelegenheit erwähnt er auch den konkreten Aufbewahrungsort im „Naturalienkabinette zu Barby in Chursachsen“. ${ }^{21}$ Mit merklichem Stolz berichtet er weiter: „Dieses [Krokodil] ist, besonders in Ansehung des Umfangs, bei weitem das Größte, das ich jemals in irgend einem Naturalienkabinette gesehen habe. Es maaß in der Länge beinahe sechzehn Fuß.“22 Das Naturalienkabinett in Barby gehörte zu den dortigen Einrichtungen der Brüdergemeine. Es unterstand der Aufsicht von Dozenten am Theologischen Seminar, dem Ort, wo nicht nur künftige Theologen und Lehrer ausgebildet wurden, sondern auch Juristen und Mediziner zumindest die anfängliche Un- terweisung in ihren Studienfächern erhielten. Das Kabinett ist wahrscheinlich bereits 1755 angelegt. ${ }^{23}$ Eine handliche Sammelanweisung des damaligen Verantwortlichen, Johann Jacob Bossart (1721-1779), zum Gebrauch der Missionare, die die hauptsächlichen Einlieferer darstellten ${ }^{24}$, erschien 1774 im Druck. ${ }^{25}$ Inventarverzeichnisse liegen ab diesem Jahr vor und zeigen nicht so sehr ein großes Interesse an „Kuriositäten“, sondern unterstreichen vielmehr die naturwissenschaftlichen und ethnographischen Interessen der Herrnhuter. In der Tat stößt man im Katalog der „Kunst-Sachen“26 auf 17 Nummern, die entweder direkt aus Ägypten stammten oder über Ägypten aus dem Orient nach Barby gelangt waren. Je vier Objekte wurden von John Antes und Georg Heinrich Wieniger (1745-1815) eingeliefert. Bei den übrigen Objekten fehlt eine Angabe des Sammlers. Leider existiert das Kabinett nicht mehr. Mit der Aufgabe der Herrnhuter Niederlassung in Barby zog es 1808 nach Niesky um, wobei übrigens die Beine des unter einen Wagen gebundenen Krokodils auf dem Wege verloren gegangen waren. ${ }^{27}$ In Niesky wurde das „Museum“ zum Großteil bei Ende des Zweiten Weltkrieges zerstört. Reste des ehemaligen Kabinetts sind jedoch in das Völkerkundemuseum nach Herrnhut gelangt, wo immerhin noch acht Katalognummern aus Ägypten identifizierbar sind. Besondere persönliche Bedeutung für Antes hatten ein Strick zum Halten der Füße und eine sogenannte „Korbatsche“, d.h. ein „Stock aus der Haut des Nielpferdes geschnitten, womit die Araber bey Cairo dem Br. John Antes Schläge auf die Fußsohlen gaben.“ Das „Kosten“ dieser Ethnographica beschrieb Antes sehr ausführlich in seinen Beobachtungen. Der Hergang ist kurz dieser:

„Jeder von den Leuten, die den Stock mit der Kette fassen, vermittelst dessen die Füße so in die Höhe gehalten werden, daß sie eine horizontale Stellung haben, hält eine Korbatsche in der Hand, womit sie auf Befehl ihrer Herren wechselsweise wie Drescher darauf los schlagen. Wenn jemand die Bastonade bekommt, so sagt man, daß er die Korbatsche erhält, manchmal aber auch, daß er dieselbe kostet.“ ${ }^{29}$

Die Bastonade bescherte ihm ein lang anhaltendes schmerzhaftes Andenken.

Dass der als Geigenbauer, Uhrmacher und Erfinder ${ }^{30}$ mechanisch und als Komponist ${ }^{31} \mathrm{mu}$ sisch interessierte Antes gerade ein fein verziertes Instrument, vermutlich eine arabische Kamanche (Stachel- oder Spießgeige) mitbrachte, darf nicht verwundern. Der Tischler Wieniger brachte ein Jahr später (1783) neben einem Paar verzierter Stelzsandalen für Frauen (arabisch: qabqab) ${ }^{32}$ vor allem Gegenstände des alltäglichen religiösen Gebrauchs (Kreuz, ,Rosenkränze“) mit. Um als Mitbringsel 1780 im
John Antes (1740-1811), 1770-1782 Uhrmacher in Ägypten, um 1785

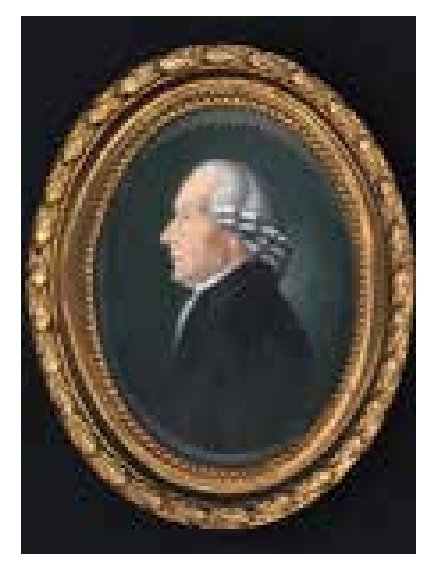

Georg Heinrich Wieniger (1745-1815) 
Unbekannter Künstler: Ruine "Alt-Cairo" am Heinrichsberg in Herrnhut, 1773 errichtet von Samuel Roller, um 1775 Unitätsarchiv Herrnhut, TS Mp.12.2
Halsfragment einer Kamanche (Stachel oder Spießgeige), gesammelt von John Antes, vor 1782

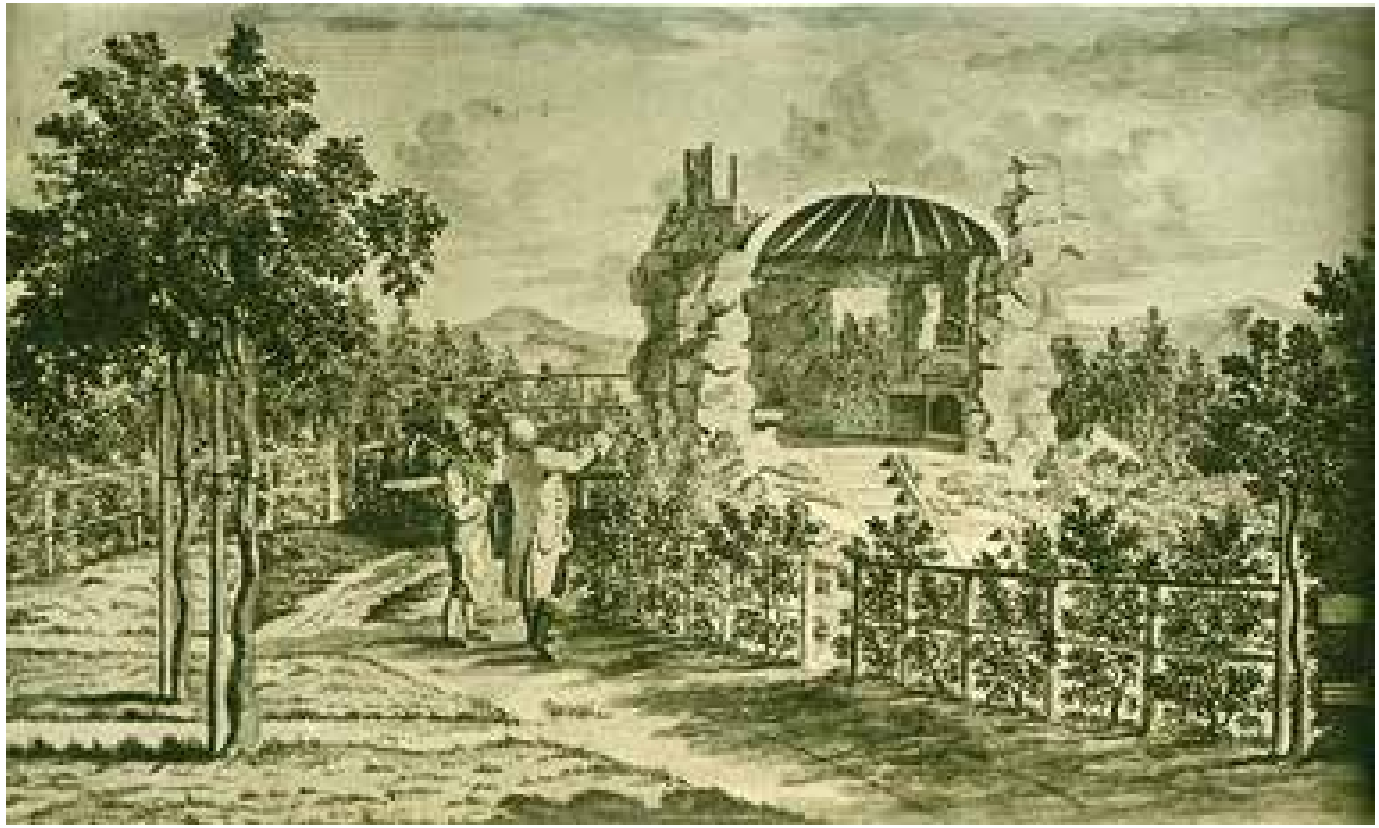

Besitz Schachmanns gewesen zu sein, kamen Antes und Wieniger als Überbringer des türkischen Schreibzeugs allerdings zu spät aus Ägypten. Man wird daher entweder an eine ältere Erwerbung von Hocker oder eine Zusendung auf dem „Postwege“ denken müssen.

Es zog nicht nur Hocker immer wieder, letztmals 1767, nach Kairo. Insgesamt wurde etwa ein Dutzend Herrnhuter nach Ägypten entsandt, um unter den koptischen Christen zu wirken; die Hoffnung auf eine Mission unter den Nichtchristen hatte man aufgeben müssen. Augustin Samuel Roller, von Beruf ein Chirurgus, träumte über viele Jahre von einem Einsatz in Ägypten; sicherlich nicht zuletzt angeregt von Hocker, mit dem er zusammen im Haus der ledigen Brüder lebte, wenn Hocker in Herrnhut war. Seine Sehnsucht war so groß, dass er 1773 am Heinrichsberg in Herrnhut eine Ruine erbaute bzw. erbauen ließ, „Alt Cairo“ genannt. Heute gibt nur noch die Reproduktion eines verlorenen Aquarells einen bildlichen Eindruck

von dem Bauwerk, dass ansonsten verschwunden ist. Besucher der 1770er Jahre wie Adolf Traugott von Gersdorf ${ }^{33}$ oder der Graf Friedrich Ulrich zu Lynar berichten davon in ihren Reisetagebüchern; letzterer erblickte darin „eine Nachahmung von Alt-Cairo. Das Haus aber sieht mehr einer eingefallenen Hütte, als den Ueberbleibseln alter Pracht ähnlich. Ein empfindsamer Chirurgus Namens $===$, fühlte in Herrnhut einen Trieb nach Alt-Cairo, der endlich so dringend war, daß er dieses Monument erbauete: jetzt befindet er sich in dem wirklichen alten Cairo.“34

Doch noch einmal zurück zu Schachmann selbst. Ähnlich wie Roller fühlte er sich nach Ägypten hingezogen. Als Roller und Wieniger ihren Ruf nach Ägypten erhielten, äußerte er sich „wie ihm nicht lieber seyn könte, als wenn er von der U[nitäts] $\ddot{A}[$ ltesten]C[onferenz] einen Auftrag erhielte, den Bruder Roller und seinen Gefehrten nach Cairo zu begleiten. Er hoffe nicht nur, diesen Brüdern auf ihre Reise mit der

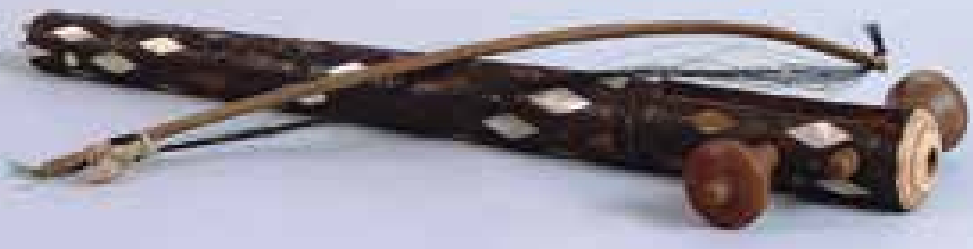
französischen und italiänischen Sprache nüzlich dienen zu können, sondern verspreche sich auch davon einen eigenen Segen für sein Herz und seinen in so mancherley Connexionen verwickelten Gang, von welchen er bey Gelegenheit einer langen Reise am ersten loszukommen hoffe." ${ }^{35}$

Doch die Kirchenleitung, die von dieser Äußerung Schachmanns in Kenntnis gesetzt worden war, ahnte, dass manche „Neben-Abschichten“ im Spiel seien: „ob das Anerbieten des Bruders Schachmann von allen Neben-Absichten befreyet, und ob nicht die Begierde Italien und allerley Antiquitaeten und andere Seltenheiten zu sehen damit verbunden sey. Überdem äuserte man sich, daß er wohl ein leichteres und wirksamers Mittel wählen könte, sich seiner vielen 
Welt-Bekantschaften zu entschlagen, wenn er nemlich öfter und länger in Herrnhuth seinen Aufenthalt näme. “36

Nichts desto trotz versicherte sich die Kirchenleitung durch den seinerzeit üblichen Gebrauch des Loses von dem göttlichen Willen in dieser Frage. Das Los war negativ und so wurde aus Schachmanns Reise in den Orient nichts. Die Neugier und das kunstgeschichtliche Interesse Schachmanns, die ihn wohl von Jugend an begleitet hatten, teilten seine Zeitgenossen innerhalb der Brüdergemeine kaum, wie an dem Bericht über einen Besuch der Cheops-Pyramide bei Gizeh, die von den Herrnhutern immerhin aufgesucht wurde, deutlich wird:

„Des folgenden Tages früh ritten wir zu denen 2 grossen Piramiden 11/2 Meilen von Gisa. In die gröste und renommirteste krochen wir hinein. Der erste Gang, der etwa 50 Schritt lang ist und von Norden nach Süden schräg hinunter geht, wurde am Ende so enge, daß ich mir nicht traute durchzukommen. Bruder C[ossart] aber, der hinter mir drein kam, encouragirte mich sehr, ihm wacker voranzugehn, welches ich denn that und mit genauer Noth durchkam. Dann führte uns ein andrer Gang Westwärts in die Höhe in einen kleinen Saal, dessen Fuß-Boden ein purer Stein-Haufe und die Einwohner Fledermäuse waren. Von hier stiegen wir weiter hinauf in den obersten Saal, in welchem ein fast unausstehlicher Dampf war. Nachdem wir uns ihn ein wenig mit brennenden Wachskerzen besehen hatten, sungen wir den Vers: Herr Ze-

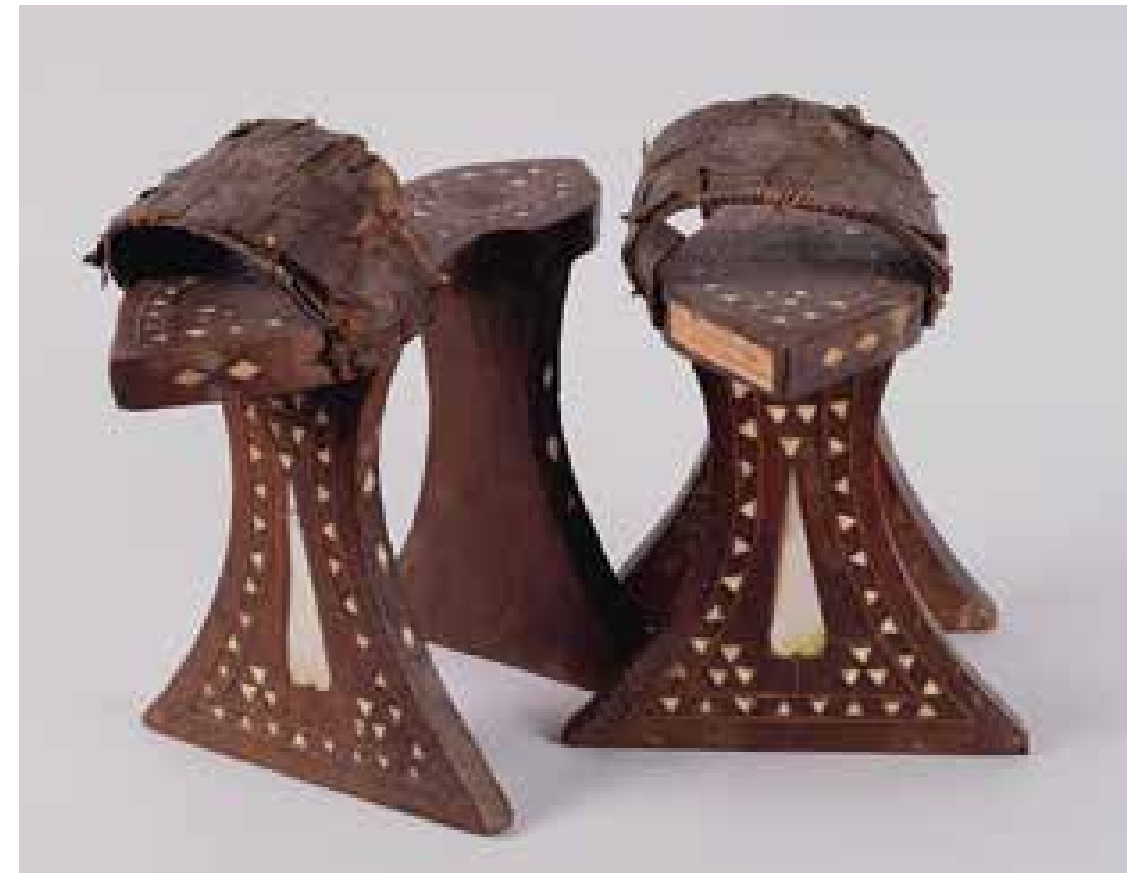

baoth, du wahrer Gott etc. Alsdenn kehrten wir bald wieder um und waren sehr erfreut, daß wir aus diesem fürchterlichen Monument wieder in die freye Luft kamen; und so langten wir gegen Abend wieder in Cairo an.“" ${ }^{37}$

In Schachmanns Kupferstichsammlung sucht man vergeblich nach einer Abbildung von ägyptischen Altertümern. Wahrscheinlich waren ihm bereits kursierende Abbildungen künstlerisch zu dürftig und zeigten einen zu unbefrie-
Stelzsandalen (qabqab), gesammelt von Georg Heinrich Wieniger (1745-1815), um 1810

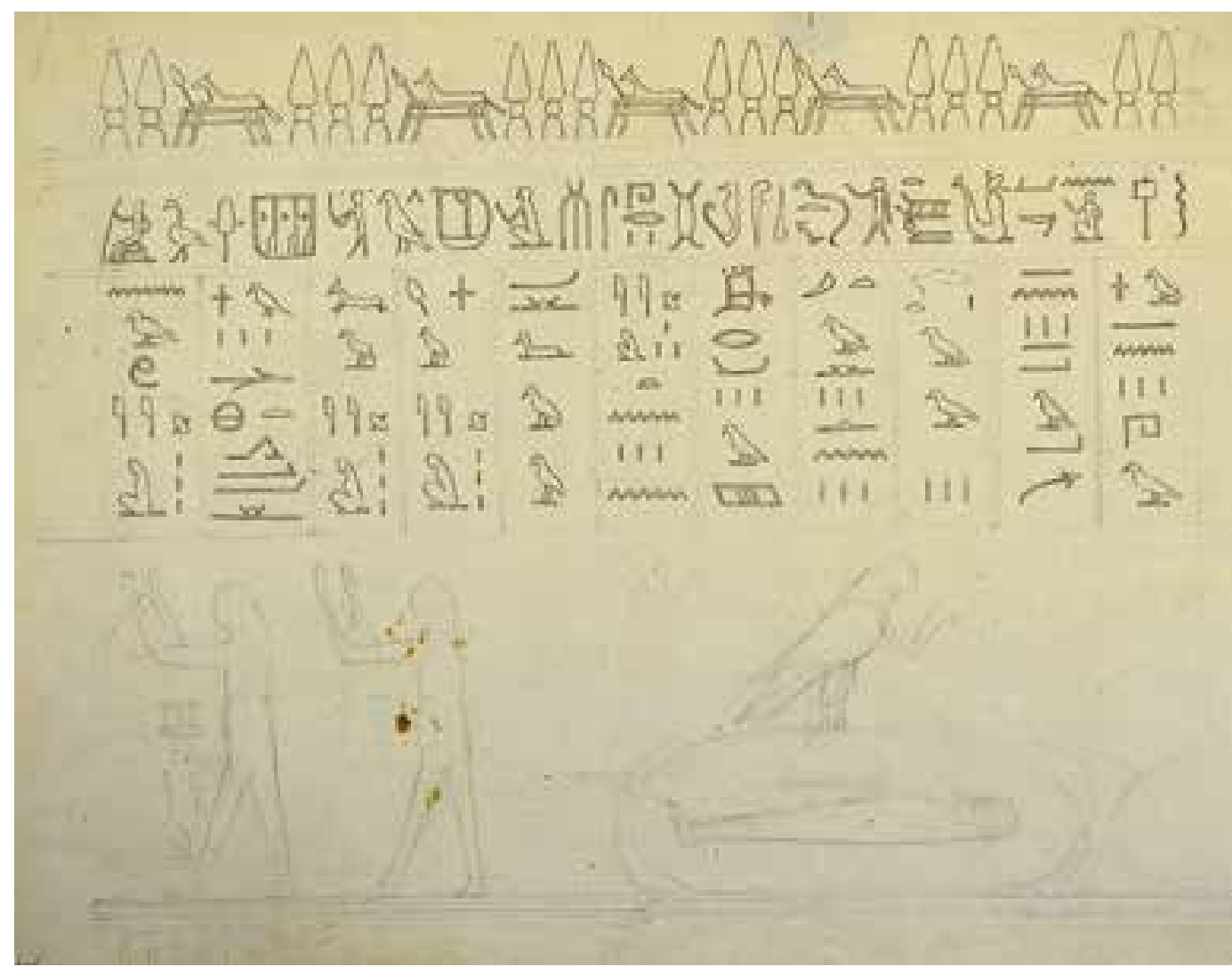

Carl Adolph Gottlob von Schachmann (?): Ägyptische Hieroglyphen, Graphit Kulturhistorisches Museum Görlitz Foto: Kai Wenzel 
digenden Grad an Exaktheit, als dass sich Schachmann dazu entschließen konnte, sie zu erwerben. Allerdings ist unter seinen Zeichnungen immerhin eine Abzeichnung von Hieroglyphen vorhanden. Vermutlich handelt es sich um eine Inschrift eines der Objekte in seiner Sammlung. Die nur schwach erkennbaren Figuren auf dem unteren Teil des Blattes erscheinen links in der Haltung wie bei einer Opferhandlung, jedoch halten sie scheinbar keine Opfergaben in Händen. Rechts ist eine Mumie in einer Kartusche zu sehen, auf der der Horusfalke sitzt. Genaueres wird aber erst zu erfahren sein, wenn sich Spezialisten an die Identifizierung des Textes machen.

Jüngst wurde von Kai Wenzel in den Görlitzer Kunstsammlungen eine Federzeichnung des Tempels von Luxor entdeckt. ${ }^{38}$ Die auf ihr enthaltenen Farbangaben sowie Detailstudien zweier Köpfe zu Staffagefiguren sprechen dafür, dass die Zeichnung vor Ort entstand. Der Quadratur nach zu schließen, sollte es in ein vergrößertes Gemälde umgesetzt werden. Dies lässt nun doch noch an eine eigene Orientreise Schachmanns denken. Im Spätsommer 1775 nahm Schachmann bei drei Gläubigern zusammen 1100 Taler auf. Anschließend klafft bisher eine Lücke von mehreren Monaten bis Mitte 1776 in seinem Itinerar.

$1 \mathrm{Zu}$ Schachmann siehe v.a. [Schmidt, Christian Samuel: Nachruf auf] Karl Adolph Gottlob von Schachmann. In: Lausitzisches Magazin, Viertes Stück, vom 28ten Februar, 1789, S. 55-59; Denkschrift, auf Herrn Karl Adolf Gottlob von Schachmann auf Königshain und Oberlinda. In: Lausitzische Monatsschrift 1793. April. Viertes Stük, S. 200-212 und Mai. Fünftes Stük, S. 257-273; Otto, G. F.: Lexikon der seit dem 15. Jahrhundert verstorbenen und jetztlebenden Oberlausitzischen Schriftsteller und Künstler, 3. Band, Görlitz 1803, S. 125-127; Reuß, Eleonore Fürstin: Carl v. Schachmann. Ein Bild aus dem geistigen Leben des 18. Jahrhunderts. In: Allgemeine konservative Monatsschrift für Politik, Literatur und Kunst, 54 (1897), S. 33-45, 171-181; Orphal, Horst: Karl Adolph von Schachmanns Leben und Apologie für Zinzendorf. In: Unitas Fratrum, 9 (1981), S. 70101, und Lemper, Ernst-Heinz: Carl Adolph Gottlob von Schachmann. Zittau 2001.

2 „Historie Anc[ienne] Des Egyptiens, [Des] Carth[aginois], [Des] Assyriens, [Des] Babyloniens, Des Mèd[es] [Et des] Pers[es], [Des] Maced[oniens], [Des] Grècs. Par M. [Charles] Rollin. Amsterdam 1733 \{6 Bd.\}“ „Recherches philosophiques sur les Egyptiens et les Chinois. Par Mr. [Cornelius] de P[auw], Berlin 1773. T. I. II." (Verzeichniß der zum Familien-Fiedeicommiß und Majorats-Gute Konigshayn Ober und Niedern Antheils gehörigen Bibliothek: SStA - StFilA Bautzen, 50156 - Gutsherrschaft Königshain, 233, fol. 102-118; hier fol. 111v und 117r).

3 Zu Desneval siehe Kjølsen, Frits Hammer: Roko- kogreven Pierre d'Esneval og Christian VIs etiopiske projekt. Aarhus 1968; ergänzend dazu Manukyan, Artur: Konstantinopel und Kairo - Die Herrnhuter Brüdergemeine im Kontakt zum Ökumenischen Patriarchat und zur Koptischen Kirche. Interkonfessionelle und interkulturelle Begegnungen im 18. Jahrhundert. Würzburg 2010 (Orthodoxie, Orient und Europa, 3) [zugl. Hochschulschrift: Göttingen, Univ., Theol. Fak., Diss. 2009], S. 212-221.

4 Tagebuch der Sophie Auguste von Schrautenbach, zum 28. Juni 1752 (Unitätsarchiv Herrnhut, künftig: UA, R.21.A.160).

$5 \mathrm{Zu}$ den Aktivitäten der Herrnhuter im osmanischen Reich siehe Makukyan, Konstantinopel und Kairo (wie Anm. 3).

6 Manukyan, Konstantinopel und Kairo (wie Anm. 3), S. $220 \mathrm{f}$.

7 „There being no Liturgys[!], I \& Henrich [XXVIII. Reuß] proposed a walk, \& took Nicolas Watteville \& Charles a long, we went thro the Herrschafts Garten, down in the Valey \& so to the 7 Meadows; [...]. in the 7 Meados we found Hoker \& Luedeke; \& diverted us on the lovely walk the like is not hereabouts but in these Meadows. We disc[oursed] much of Count Desneval, \& Hoker related odd Stories of him." (Tagebuch Heinrich XXXI. Reuss gen. Ignatius, zum 9. Mai 1756: UA, R.21.A.146. IV).

8 Tamcke, Martin / Manukyan, Arthur (Hg.): Herrnhuter in Kairo. Die Tagebücher 1769-1783. Göttingen 2012. (Orthodoxie, Orient und Europa, Bd. 5 = Herrnhuter Quellen zu Ägypten, Bd. 1), S. 58

9 Vgl. Philipp, Guntram: Integrationsprobleme im 18. Jahrhundert. Ein Türke am Weimarer Hofe und bei den Herrnhutern. In: Pietismus und Neuzeit, 33 (2007), S 99-127.

10 Tagebuch Heinrich XXXI. Reuß gen. Ignatius, zum 3. Oktober 1755 (UA, R.21.A.146.III).

11 Hoker inviting us to a Turkish, i.e. very good Coffee; he dressed himself in his best turkish Cloaths, \& his Doctors-Cap; Charles in his second best, \& had einen Schnurrbart; \& Benny dressed himself in the remains of Hokers Cloaths; Charles lookd' grand \& very fine, the full \& good Face \& short Neck belonging to the turkish beautifull Dress, but his Whiskers made so changed his Face, that manny Br[etheren] could not tell for their Lives, who it was; \& Frederic Watteville did not know him at all; a great assembly then met in Andresens room, tasted the turkish Coffee; \& Benny was sadly plagued he cutting an odd Figure, \& Hoker calling him a Turk, which will become a Christian. Hoker then went about in the house in all the rooms \& made a great Joy among the Br[etheren], some Br[etheren] got up from their places, frightend, \& took of their Caps for the strange Gentleman, whom they after looking narrowly, knew very well. (Tagebuch Heinrich XXXI. Reuß gen. Ignatius, zum 23. April 1756 : UA, R.21.A.146.IV).

12 CATALOGUE RAISONNÉ D'UNE COLLECTION DE MÉDAILLES. à LEIPZIG, de l'Imprimerie de B[ernhard] C[hristoph] Breitkopf \& Fils [1774].

13 Vgl. Inventar Dresden-Antikensammlung (1820/ 33), fol. 34v: Nr. 152: Isis, die den Horus säugt, Bronze. Inventar Dresden-Antikensammlung (1765) - Reinschrift. SStA - HStA Dresden, Kunstsammlungen Dresden - Inventare, 495 (= SK Dresden, Mikrofilm 80): Isis mit Horus, Bronze auf schwarz gebeiztem hölzernen, runden Postament, Höhe: 51/2 Zoll. (F.A.12.) [fol. 93r]; Isis averrunca, a. weiß-grünliches Porzellan, Höhe: 4 Zoll, b. türkisblaues Porzellan; Höhe: 31/2 Zoll, c. 
türkisblaues Porzellan; Höhe: 3 Zoll (F.A.13-15) [fol. 146v].

14 Johann Friedrich Wacker an Carl Adolph Gottlob von Schachmann. Dresden, 10. Februar 1765. OLB Görlitz, Nc III 533 [\#2]. Wacker gedenkt im Kontext der Numismatik 1767 Hockers: „Dem charmanten Herrn Doctor Hocker wüntsche eine glückliche Reise." (Wacker an Schachmann. Dresden, 16. März 1767; ebd. [\#4]).

15 Leske, Nathanael Gotfried: Reise durch Sachsen in Rüksicht der Naturgeschichte und Ökonomie. Leipzig: J. G. Müllersche Buchhandlung, 1785, S. 171.

16 Ebd.

17 BEOBACHTUNGEN UEBER DAS GEBIRGE BEY KEOENIGSHAYN IN DER OBERLAUSITZ.| DRESDEN, 1780. In der WALTHERISCHEN Hofbuchhandlung.

18 Anzeige von den Samlungen, II (1781), S. 43.

19 Beobachtungen über den Orient aus Reisebeschreibungen. Dritter Theil welcher die Zusätze der neuen und vermehrten englischen Ausgabe enthält. Aus dem Englischen. Hamburg bey Carl Ernst Bohn. 1779, S. 480 (nach Schulzens Reisen, $\mathrm{V}, 330 \mathrm{ff}$.).

20 Antes, John: Beobachtungen über die Sitten und Gebräuche der Aegypter, über die Nilüberschwemmung und ihren Einfluß, nebst Bemerkungen über die Pest und andere Gegenstände während eines zwölfjährigen Aufenthaltes zu Kahira und in seiner Nachbarschaft niedergeschrieben. Aus dem Englischen mit Anmerkungen übersetzt. Gera und Leipzig: Wilhelm Heinsius der Jüngere, 1801, S. 72

21 Ebd., S. 77.

22 Ebd.

$23 \mathrm{Zu}$ den Anfängen der Kunst- und Naturaliensammlungen in der Brüdergemeine siehe Augustin, Stephan: Vom Naturalienkabinett zum Völkerkundemuseum. Zur Geschichte ethnographischen Sammelns innerhalb der Evangelischen BrüderUnität (Herrnhuter Mission). In: Jahrbuch des Museums für Völkerkunde zu Leipzig, 41 (1997), S. 81-89; ders.: Das Naturalienkabinett in Barby - Anfänge des naturkundlichen und völkerkundlichen Sammelns in der Evangelischen Brüder-Unität. In: Unitas Fratrum, 55/56 (2005), S. 1-16; ders.: „Vielen einen Genuß und Belehrung gewähren und unseren Nachkommen einen Schatz bewahren“. 125 Jahre Völkerkundemuseum Herrnhut - Zur Geschichte des Museums. In: Abhandlungen und Berichte der Staatlichen Ethnographischen Sammlungen Sachsen, Band 52. Berlin 2005, S. 275-296.

24 Hinsichtlich der Zahl Objekte wie auch ihres Wertes für die Ethnographie stellt die durch Benjamin La Trobe gestiftete Sammlung von Cooks Reisen die bedeutendste Einzelzuwendung unter den „Kunstsachen“ dar. (Vgl. Nippa, Annegret (Hrsg.): Ethnographie und Herrnhuter Mission. Völkerkundemuseum Herrnhut. Katalog zur ständigen Ausstellung im Völkerkundemuseum Herrnhut, Außenstelle des Staatlichen Museums für Völkerkunde Dresden. Red.: Petra Martin und Stephan Augustin. Dresden 2003, S. 18-25; Augustin, Stephan: James Burney und seine Sammlung im Naturalienkabinett Barby. In: James Cook und die Entdeckung der Südsee. Kunst- und Ausstellungshalle der Bundesrepublik Deutschland, Bonn, 28. August 2009 bis 28. Februar 2010; Museum für Völkerkunde, Wien, 10. Mai bis 13. September 2010, Historisches Museum Bern, 7. Oktober 2010 bis
13. Februar 2011. Hrsg.: Kunst- und Ausstellungshalle der Bundesrepublik Deutschland GmbH Übers. aus dem Engl.: Eva Ambros. München 2009, S. 69 f.).

25 Bossart, Johann Jacob: Kurze Anweisung Naturalien zu samlen. Barby 1774. Vgl. Nippa, Ethnographie und Herrnhuter Mission (wie Anm. 26), S. 16 f.

26 So die Bezeichnung auf dem Etikett des Einbandes; nach Titelblatt: [Bossart, Johann Jacob: Catalogus der] Kunst-Sachen [des Naturalienkabinetts in Barby, begonnen 1775]: UA, CIM V.5.

27 Augustin; Stephan: Genuß und Belehrung (wie Anm. 25), S. 16.

28 Antes, Beobachtungen (wie Anm. 22), S. 111122, 126-129, vgl. auch S. 77f.

29 Ebd., S. 126.

30 Vgl. Selch, Frederick R.: Moravian musicians and musical instrument makers in early America and the influence on them of the Saxon-Vogtländisch industry of musical instrument making. In: Journal of the Violin Society of America 13 (1992), 1, S. 161-194; Connor, Margaret: John Antes as craftsman and inventor 1740-1811. In: Moravian History Magazine, 22 (September 2002), S. 2327.

31 Vgl. Knouse, Nola Reed (Hg.): Biographical sketches. The Music of the Moravian Church in America. Rochester, NY 2008, S. 267-289.

$32 \mathrm{Vgl}$. Graf ohne Grenzen. Leben und Werk von Nikolaus Ludwig Graf von Zinzendorf. Ausstellung im Völkerkundemuseum Herrnhut, Außenstelle des Staatlichen Museums für Völkerkunde Dresden, und im Heimatmuseum der Stadt Herrnhut vom 26. Mai 2000 bis zum 7. Januar 2001. Konzeption: Paul Peucker, Stephan Augustin, Wolfgang Langerfeld. Katalogred.: Dietrich Meyer, Paul Peucker, Karl-Eugen Langerfeld. Bildred.: Esther von Ungern-Sternberg. Herrnhut 2000, S. 198 (Kat.Nr. 244)

33 „Wenn man aus diesem Lustwalde von allerley Sorten lebendigen Holzes herauskommt, kommt man auf einen angenehmen Platz von lauter kleinen Gärten, welche den Kindern in der Anstalt gehören. Der lezte wird ein kleiner botanischer Garten und gehört Herrn Roller, dem hiesigen geschickten Chirurgo. Hier ließ selbiger von den hier befindlichen großen Steinen Ruinen aufführen." (Reisejournal A. T. von Gersdorf, zum 14 Mai 1773, OLB, ATvG 61 = Bd. 2, S. 36).

34 Des Grafen F. U. zu Lynar auf Schloß Lübbenau Journal einer Lustreise in die Oberlausitz, im November 1777. In: Johann Bernoulli's Sammlung kurzer Reisebeschreibungen und anderer zur Erweiterung der Länder- und Menschenkenntniß dienender Nachrichten. Band 1, Berlin/Altenburg 1781, S. 159-196, hier S. 172.

35 UA, UAC-Prot 1774/II, S. 88 f., zum 15. April 1774.

36 Ebd.

37 Diarium von Pilder (und Cossart an Johannes von Watteville), 9. Juni bis 5 . Juli 1758 , zum 4 . Juli (UA, R.17.B.6.a.1758.q). Der zitierte Liedvers Zinzendorfs aus dem Jahr 1742 lautet: „HErr Zebaoth, du wahrer Gott | der creatur, GOtt Schöpfer der natur, | GOtt, der die ganze welt erhält, | und was verdarb mit blut erwarb, und heiligt all's ! dir falln wir um den hals." (Herrnhuter Gesangbuch, Nr. 1812, Vers 7).

38 „Ruins at Luxor over against Carnac 135 Legues[?] from Cairo" (StKS Görlitz, OLH 18 - unverzeichnet). Ich danke Kai Wenzel für Hinweis und Expertise zu seinem Fund.

\section{Autor}

Dr. Rüdiger Kröger

Langenhagen 